\title{
Eficácia das transposições verticais dos músculos retos horizontais nas anisotropias alfabéticas
}

\author{
The effectiveness of vertical transpositions of the horizontal rectus muscles in $A$ and \\ V pattern strabismus
}

Marcelo F. Gaal Vadas ${ }^{(1)}$

Carlos R. Souza-Dias (2)

Carlos F. Uesugui ${ }^{(3)}$

\section{RESUMO}

Objetivo: Transposição vertical dos músculos retos horizontais é a técnica de eleição para as anisotropias verticais em que não há disfunção de músculos oblíquos que as justifique. O objetivo foi avaliar o resultado das transposições efetuadas e identificar quais os fatores determinantes do resultado, para estabelecer-se relação que informe a magnitude da transposição que deva ser realizada para determinada magnitude de anisotropia.

Métodos: Avaliaram-se retrospectivamente 43 pacientes portadores de anisotropias em $\mathrm{A}$ ou $\mathrm{V}$, associado a estrabismos horizontais essenciais, sem disfunção de músculos oblíquos, submetidos à transposição vertical bilateral e simétrica dos músculos retos horizontais

Resultados: $81,4 \%$ dos pacientes apresentavam esotropia com forma em A. A média das anisotropias situou-se em torno das 19 DP e a maior parte das transposições foi de $4 \mathrm{~mm}$, obtendo-se 66,7 a $79,5 \%$ de sucesso, isto é, casos com anisotropias residuais em A até 5 DP ou $V$ até 10 DP. A correção da anisotropia correlaciona-se intensamente com a magnitude da anisotropia pré-operatória e com a magnitude da transposição efetuada, sobretudo com aquela, sugerindo assim ser o procedimento auto-ajustável. A equação linear com as 3 variáveis mostrou-se clinicamente incoerente e foi, portanto, abandonada.

Conclusões: A transposição vertical e simétrica dos músculos retos horizontais é eficaz para o tratamento das anisotropias verticais sem disfunção de oblíquos, sendo este resultado estável em seguimento prolongado.

Palavras-chave: Transposição; Anisotropia.

\begin{tabular}{l} 
INTRODUÇÃO \\
\hline As incomitâncias verticais, anisotropias verticais, anisotropias alfabé- \\
ticas, síndromes alfabéticas ou padrões em "A"(A) ou em "V"(V) referem- \\
se a estrabismos em que o desvio horizontal varia no eixo vertical, isto é, \\
estrabismos horizontais com magnitudes diferentes ao olhar para cima e \\
para baixo. Essa variação segue dois padrões básicos: em A ou em V. O \\
padrão A corresponde à maior proximidade dos polos anteriores dos globos \\
oculares entre si em supraversão e maior afastamento em infraversão, \\
podendo, portanto, ser uma esotropia (ET) de maior magnitude em \\
supraversão e menor em infraversão ou uma exotropia (XT) de menor
\end{tabular}

Apresentado no Congresso do Conselho Latino-Americano de Estrabismo (CLADE), Buenos Aires, 9 a 11 de maio de 1996.

(1) Médico-Voluntário do Depto. de Oftalmologia da Santa Casa de S.P. Pós graduando do Depto. de Oftalmologia do HC da Faculdade de Medicina da USP.

(2) Professor Titular da Faculdade de Ciências Médicas da Santa Casa de SP.

Professor Assistente da Faculdade de Ciências Médicas da Santa Casa de SP.

Cada autor declara que não possui interesse financeiro no presente estudo.

Endereço para correspondência: Marcelo F. Gaal Vadas. R. Calçada das Draçenas, 10. Barueri (SP). CEP 06453-000. e-mail: mvadas@ig.com.br 
magnitude em supraversão e maior em infraversão. O padrão $\mathrm{V}$ corresponde ao inverso do A. Existem descritos outros padrões de anisotropias, em X, Y, lambda e losango, que são variantes dos em A e V, menos freqüentes e encontrados principalmente nas exotropias ${ }^{14}$.

A etiopatogenia das incomitâncias verticais tem sido assunto controverso desde a década de 1950, tendo surgido, a partir de então, diversas teorias a esse respeito.

Para Urist ${ }^{1}$, a causa estaria na disfunção dos retos horizontais, partindo do princípio de que os retos mediais são mais solicitados para o olhar para perto e para baixo e os retos laterais para longe e para cima.

Brown ${ }^{2}$ sustentava a hipótese de que a causa seria primariamente a disfunção dos retos verticais e, secundariamente, dos seus sinergistas contralaterais (oblíquos), que têm função abdutora, o oblíquo inferior (OI) em supraversão e o oblíquo superior(OS) em infraversão.

Urrets-Zavalía ${ }^{3}$ advogava a teoria de que a conformação facial mongolóide predisporia à ET com hipofunção dos OI, com conseqüente forma em $\mathrm{A}$, e à XT com hiperfunção dos $\mathrm{OI}$, com conseqüente forma em $\mathrm{V}$. Na conformação caucasiana, anti-mongolóide, haveria predisposição inversa, isto é, ET com hiperfunção dos OI e portanto, forma em V e XT com hipofunção dos OI e forma em A. Atualmente, parece unânime atribuir-se o problema, na maioria dos casos, à disfunção dos oblíquos, quer seja de causa inervacional ou mecânica. Em contrapartida, e corroborando o aspecto plurifatorial das anisotropias ${ }^{14}$, encontramos, não infrequentemente, anisotropias verticais sem disfunção dos oblíquos, base de estudo deste trabalho.

Para a correção das anisotropias verticais, quando não há disfunção dos oblíquos ou quando a disfunção é discreta, desproporcional ao montante da incomitância, lança-se mão de uma técnica cirúrgica, descrita inicialmente por Knapp ${ }^{4}$ em 1959 e elaborada, subsequentemente, por Postic ${ }^{18}$, a transposição vertical dos músculos retos horizontais, simultânea, bilateral e simétrica.

A mecânica orbitária é assunto complexo, que foge do escopo deste trabalho; entretanto, algumas considerações acerca dessa técnica serão traçadas. Sabe-se que a ação muscular será simples,i.é., em torno de um único eixo de Fick, se o plano de ação do músculo (determinado pela linha de ação e centro de rotação) contiver o eixo visual, como ocorre com os retos horizontais em posição primária do olhar (PPO). Entretanto, a ação se torna complexa quando não mais há essa "congruência", como ocorre, por exemplo, em infraversão. Nesta situação, o eixo visual não coincide com o plano de ação, surgindo então novos vetores ou componentes, em detrimento da ação principal horizontal, o componente vertical e o componente torcional. Podemos incrementar esse efeito fisiológico abaixando ou elevando cirurgicamente as inserções musculares. Por exemplo, se abaixarmos as inserções anatômicas dos retos mediais, estaremos decompondo o vetor horizontal, tanto mais quanto maior o deslocamento e quanto mais para baixo se dirigir o olhar, uma vez que o eixo visual (ou o plano meridional horizontal, ou ainda, a horizontal fundamental, como preferem alguns autores -17-) afasta-se do plano de ação. Por outro lado, ao olhar para cima, o eixo visual se aproximará do plano de ação, "recompondo" o componente primário horizontal (fig.1). Portanto, a inserção do músculo deve ser transposta no sentido para o qual queremos o seu maior debilitamento horizontal. Assim, nas ET com V, retrocedem-se e abaixam-se os retos mediais e, nas $\mathrm{XT}$ com V, retrocedem-se e elevam-se os retos laterais. Analogamente, nas ET com A, retrocedem-se e elevam-se os retos mediais e nas XT com A retrocedem-se e abaixam-se os retos laterais. Esse procedimento pode ser monocular, como descrito originalmente por Goldstein ${ }^{6}$ em 1967, com bons resultados e possíveis efeitos torcionais ${ }^{13}$, ou bilateral e simétrico, foco do presente estudo.

A importância da correção cirúrgica das anisotropias verticais não é somente estética e funcional (fusão em todas as posições do olhar), mas é fator importante na estabilidade da correção do desvio horizontal. Os procedimentos cirúrgicos para a correção do desvio horizontal não modificam por si só a anisotropia existente, que permanece inalterada após a operação.

A transposição bilateral e simétrica dos músculos retos horizontais é técnica freqüentemente utilizada, porém a magnitude necessária e suficiente para a correção de determinada incomitância não está ainda suficientemente estabelecida. $\mathrm{O}$ objetivo deste trabalho é identificar as variáveis envolvidas na correção da anisotropia e estabelecer uma relação matemática entre elas, para que se possa obter o melhor planejamento cirúrgico possível. Além disso, quisemos melhor caracterizar a população submetida a tal procedimento, obter informações acerca da magnitude da transposição e com que sucesso ela é realizada.

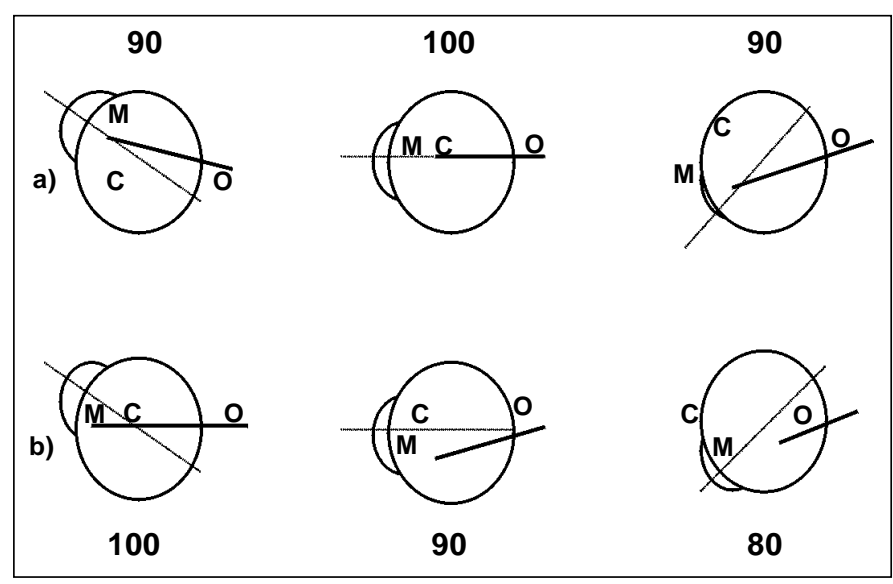

Fig. 1 - Esquema simplificado, mostrando, em secções sagitais medianas do olho, as projeções dos pontos de origem de um reto horizontal (0), do centro de rotação ocular (C) e do ponto mediano da inserção (M). a) músculo na posição original, não transposto, b) músculo transposto para baixo. Os números são valores numéricos arbitrários para a força do vetor horizontal (segundo Bicas, H.E.A. Boletim do CLADE, no.8, 1994). 


\section{MATERIAL E MÉTODOS}

Examinaram-se, retrospectivamente, prontuários de 43 pacientes portadores de estrabismo horizontal essencial associado a anisotropia vertical, sem disfunção de oblíquos, nos quais se empregou exclusivamente a técnica de retrocesso e/ ou ressecção com transposição (elevação ou abaixamento) bilateral e simétrica dos retos horizontais. Quanto à técnica cirúrgica, utilizou-se abertura da conjuntiva limbar, captura dos músculos com ganchos, ampla secção das membranas intermusculares, apreensão dos músculos com miostato, secção e reposicionamento na eclera com fio absorvível 6-0. Pacientes que tiveram também seus músculos oblíquos ou retos verticais operados foram excluídos do estudo.

Os pacientes foram selecionados do Departamento de Oftalmologia do Hospital Central da Santa Casa de São Paulo, do Centro Oftalmológico Pacaembú e da clínica particular do Prof. Carlos Souza-Dias, sendo incluídos na amostra todos os pacientes com as características acima citadas no período de junho de 1985 a dezembro de 1993.

Os dados dos pacientes foram todos tabulados, levando-se em consideração idade, sexo, refração e acuidade visual préoperatória, desvio em PPO, supra e infraversão, planejamento cirúrgico e medidas do desvio pós-operatório. Em seguida, calculou-se o montante de correção obtida para cada indivíduo em cada fase pós-operatória. Estabeleceu-se arbitrariamente como sendo pós-operatório precoce o período compreendido entre 15 dias e 6 meses, pós-operatório tardio o período entre 6 meses e 2 anos e, pós-operatório remoto, além de 2 anos após a cirurgia. Em seguida, foram selecionados, dentro de cada etapa pós-operatória, os casos satisfatórios. Consideraram-se os resultados satisfatórios anisotropia em A até 5 dioptrias prismáticas (DP) e em V até $10 \mathrm{DP}$.

As informações foram transcritas para um questionário (previamente preparado), no aplicativo Epi Info Versão 5.01, programa multitarefa, elaborado pela Organização Mundial de Saúde e "Center for Disease Control" para manusear dados epidemiológicos.

No que tange às medidas dos desvios, foram obtidas por 2 examinadores na Santa Casa e outro no Centro Oftalmológico Pacaembú, sendo todas conferidas, em última instância, pelo mesmo examinador da clínica particular (Prof. Carlos SouzaDias). Utilizou-se para a mensuração do desvio o "cover test" alternado com prisma, quando havia boa visão e fixação central em ambos os olhos, ou o método de Krimsky quando essas condições não eram satisfeitas. A anisotropia foi mensurada fixando-se um ponto de referência a 6 metros (foco luminoso), inicialmente olhando-o em frente (PPO), a seguir com o pescoço em máxima flexão para simular olhar para cima e depois em máxima extensão para simular olhar para baixo.

Foram utilizados 3 testes estatísticos: o t de Student para médias, o coeficiente de correlação e a regressão.

A idade média dos pacientes foi de 10,32 anos $\pm 8,78$ anos, variando de 10 meses a 47 anos. Predominou o sexo feminino, na proporção de $1,5: 1,0$. A média da refração foi $+2,50 \pm$ $0,25 \mathrm{DE}$ para o grupo dos hipermétropes (maioria) e $-1,50 \pm$ $0,65 \mathrm{DE}$ para o grupo dos míopes.

Dos 43 pacientes, 40 eram portadores de ET e 3 de XT. Os pacientes foram classificados de acordo com o tipo de incomitância (A e V). Houve predomínio de casos com anisotropia em A $(81,4 \%)$ e principalmente ET com A $(76,8 \%$ - Tabela 1).

Além disso, os pacientes foram separados de acordo com a magnitude da anisotropia. A média da magnitude do A foi de 18,23 DP e a do V foi de 19,75 DP, sendo a diferença entre eles não significante $(\mathrm{p}>0,05)$, como descrito na Tabela 2.

A média das transposições empregadas para grupos estratificados de anisotropias em A está demonstrado na tabela 3. A maioria das transposições foi de $4 \mathrm{~mm}(\operatorname{moda}=4 \mathrm{~mm})$, tanto para os padrões em A quanto para os padrões em $\mathrm{V}$; a amostra do grupo em $\mathrm{V}$ foi pequena.

\section{RESULTADOS}

O número de resultados cirúrgicos satisfatórios preponderou. A saber: $79,5 \%$ no período pós-operatório precoce (15 dias a 6 meses), 69,2\% no tardio ( 6 meses a 2 anos) e $66,7 \%$ no remoto (além de 2 anos).

A correção da anisotropia em A guarda relação com a

\begin{tabular}{|c|c|c|c|c|}
\hline \multirow[b]{2}{*}{ Tipo de Anisotropia } & \multicolumn{2}{|c|}{ Desvio Horizontal } & \multirow[b]{2}{*}{ TOTAL } & \multirow[b]{2}{*}{$\%$} \\
\hline & ET & $\begin{array}{l}\mathbf{X T} \\
\end{array}$ & & \\
\hline $\mathrm{A}$ & 33 & 2 & 35 & 81,4 \\
\hline V & 7 & 1 & 8 & 18,6 \\
\hline TOTAL & 40 & 3 & 43 & 100,0 \\
\hline
\end{tabular}

\begin{tabular}{|c|c|c|c|}
\hline \multirow{2}{*}{$\begin{array}{l}\text { Magnitude em } \\
\text { dioptrias prismáticas }\end{array}$} & \multicolumn{2}{|c|}{ Tipo de anisotropia } & \multirow[b]{2}{*}{ TOTAL } \\
\hline & A & V & \\
\hline $5-9$ & 3 & - & 3 \\
\hline $10-14$ & 8 & 2 & 10 \\
\hline $15-19$ & 12 & 1 & 13 \\
\hline $20-24$ & 4 & 1 & 5 \\
\hline $25-29$ & 4 & 3 & 7 \\
\hline $30-34$ & 1 & 1 & 2 \\
\hline $35 e+$ & 3 & - & 3 \\
\hline Total & 35 & 8 & 43 \\
\hline
\end{tabular}




\begin{tabular}{|c|c|c|c|}
\hline \multicolumn{4}{|c|}{$\begin{array}{l}\text { Tabela 3. Médias da magnitude das transposições empregadas, } \\
\text { em mm, para grupos de pacientes com anisotropia em A - São } \\
\text { Paulo - 1985-1993 }\end{array}$} \\
\hline \multicolumn{2}{|l|}{ Anisotropia } & \multicolumn{2}{|c|}{ Transposição } \\
\hline $\begin{array}{l}\text { Magnitude em } \\
\text { dioptrias prismáticas }\end{array}$ & $\overline{\text { Freq }}$ & Média (mm) & Desvio Padrão \\
\hline $5-9$ & 3 & 3,33 & 0,57 \\
\hline $10-14$ & 8 & 3,87 & 0,64 \\
\hline $15-19$ & 12 & 4,08 & 0,51 \\
\hline $20-24$ & 4 & 4,50 & 0,57 \\
\hline $25-29$ & 4 & 5,25 & 0,95 \\
\hline $30-34$ & 1 & 5,00 & - \\
\hline $35-39$ & 2 & 5,50 & 0,70 \\
\hline 40 ou + & 1 & 7,00 & - \\
\hline
\end{tabular}

magnitude do desvio prévio em todas as fases avaliadas $(\mathrm{r}=0,78$ para $\mathrm{n}=24$ - precoce $-; \mathrm{r}=0,76$ para $\mathrm{n}=20-$ tardio $-\mathrm{e}$ $\mathrm{r}=0,70$ para $\mathrm{n}=15$ - remoto - Gráfico 1) e, de forma menos intensa, com a magnitude da transposição empregada $(\mathrm{r}=0,59$ para $n=26 ; \mathrm{r}=0,71$ para $\mathrm{n}=19$ e $\mathrm{r}=0,62$ para $\mathrm{n}=13$ Gráficos 2). A correção da forma em $\mathrm{V}$ mostrou forte índice de correlação apenas com a magnitude do desvio prévio no período tardio $(\mathrm{r}=0,98)$.

Tentamos obter, através de regressão estatística no Epi Info, uma equação que pudesse prever a quantidade de correção da anisotropia, quando fornecidos o desvio prévio à cirurgia e a magnitude da transposição. A equação se mostrou linear somente quando continha duas das três variáveis. A equação que continha todas as variáveis (desvio prévio, magnitude da transposição e montante de correção obtida) mostrou-se clinicamente incoerente e foi, portanto, abandonada.

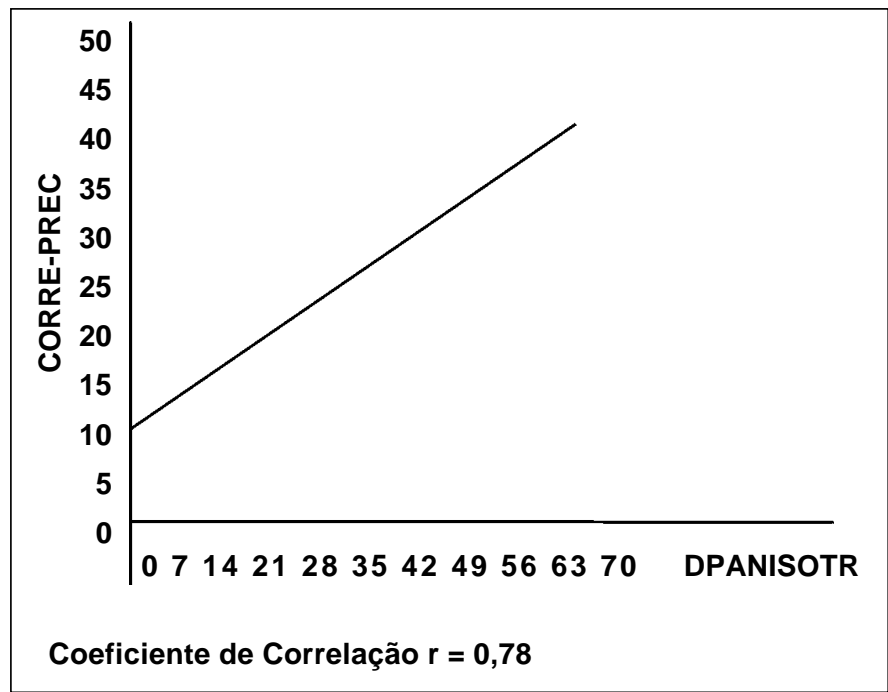

Gráfico 1 - Magnitude da anisotropia (DPANISOTR) em A segundo correção obtida na fase precoce (CORRE-PREC), ambas em dioptrias prismáticas, em 24 pacientes - São Paulo - 1985 a 1993

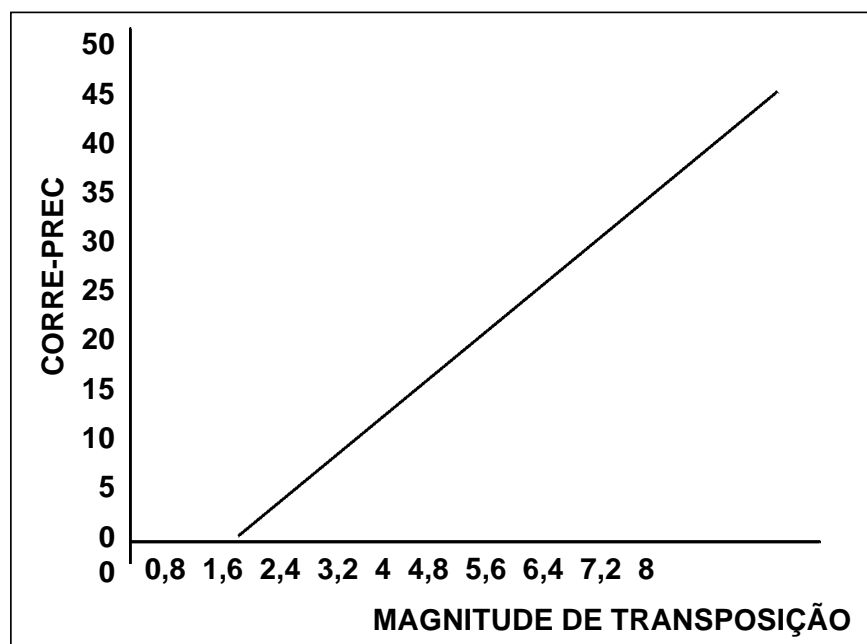

Coeficiente de Correlação $r=0,59$

Fonte: Depto. de Oftalmologia da F.C.M da Santa Casa SP; COP e consultório particular.

Gráfico 2 - Magnitude de transposição (em $\mathrm{mm}$ ) segundo correção obtida na fase precoce(em dioptrias prismáticas), em 26 pacientes com anisotropia em A - São Paulo - 1985-1993

\section{DISCUSSÃO}

A prevalência dos diversos tipos de anisotropias com ou sem disfunção de oblíquos na população de estrábicos é bastante variável. Segundo dados da literatura, representam 12,5 a $34,7 \%$. Arthur ${ }^{16}$, por exemplo, diz representarem $25 \%$ da população de estrábicos. No que diz respeito ao tipo de anisotropia, há unanimidade quanto à maior prevalência de formas em V. Segundo Villaseca ${ }^{7}$, estas representam 63,5\% dos anisotrópicos com ou sem disfunção de oblíquos e, para Almeida e Pereira Real ${ }^{8}, 72,2 \%$. Surpreendentemente, nossa amostra, composta exclusivamente de casos sem disfunção de oblíquos, mostrou $81,4 \%$ de formas em A.

Obtivemos em torno de $70 \%$ de resultados satisfatórios, com a maioria das incomitâncias pré-operatórias situando-se em torno das 19 DP e a maioria dos planejamentos cirúrgicos consistindo de transposição de $4 \mathrm{~mm}$. Essa taxa poderia ser maior se tivéssemos optado por critérios de sucesso mais amplos (V até 15 DP e A até 10 DP, por exemplo). Na nossa amostra, a transposição efetuada não criou ou modificou desvios verticais (hipertropias iatrogênicas).

A diferença no número de pacientes em cada fase pósoperatória ocorre pela não uniformidade e inconstância dos dados do seguimento pós-operatório, isto é, havia prontuários com dados referentes somente ao período precoce, outros com dados somente do período tardio e assim por diante.

Os nossos resultados assemelham-se aos obtidos por outros autores. A pequena variação (erros variáveis) que ocorre quanto aos resultados, pode ser atribuída à imprecisão da mensuração e variações de técnica cirúrgica. Por exemplo, se não tomarmos o cuidado de seccionar amplamente os 
ligamentos intermusculares e as ligações fibroelásticas existentes, não se produzirá o efeito desejado, já que os corpos musculares não deslizarão verticalmente na mesma medida que o fizeram suas inserções. Recente trabalho feito no "Smith Kettlewell Eye Research Institute - San Francisco" 12 demonstra, através de ressonância magnética, que a posição dos corpos dos músculos cujas inserções foram transpostas alterase substancialmente menos do que seria de esperar, fato atribuído ao efeito restritivo de tecidos conectivos e gordura intraorbitária. As diferenças anatômicas individuais também colaboram para essa variação. As já mencionadas membranas intermusculares, por exemplo, ora mostram-se frágeis e delicadas, ora espessas e abundantes, aumentando, neste caso, a "aderência" do músculo às estruturas vizinhas. As variações de resultados podem também ser devidas à possível variação fisiopatogênica das anisotropias.

Feitas essas considerações, citaremos os resultados de alguns autores. Scott e cols ${ }^{10}$ obtiveram $78 \%$ de correção (resultados finais consistindo de anisotropias $<10 \mathrm{DP}$ ) com a elevação ou abaixamento dos retos mediais de meio inserção ( $5 \mathrm{~mm})$, em 59 pacientes Von Noorden ${ }^{5}$ considera eficientes as transposições de 5 a $8 \mathrm{~mm}$. Já Arthur ${ }^{16}$ considera ser eficaz transposição de $5 \mathrm{~mm}$ para $20 \mathrm{DP}$ de anisotropia e transposição de $3 / 4$ de inserção $(7,5 \mathrm{~mm})$ para anisotropias maiores que $30 \mathrm{DP}$.

A forte correlação entre o montante de correção do A e a magnitude da anisotropia pré-operatória foi também verificada recentemente por Del Monte e cols ${ }^{11}$, em níveis semelhantes. Podemos depreender destas informações que o resultado cirúrgico, nas formas em $\mathrm{A}$, pode ser considerado auto-ajustável.

$\mathrm{O}$ grupo da anisotropia em $\mathrm{V}$ não mostrou correlação, na maioria dos casos, provavelmente por ser a amostra pequena. A forte correlação entre a magnitude da forma em $\mathrm{V}$ préoperatória e a correção obtida na fase tardia pode ser atribuída ao acaso $(n=4)$.

Os dados apresentados sugerem existir outras causas para as anisotropias que não somente as disfunções de oblíquos. Estas causas não são puramente mecânicas, já que o resultado relaciona-se mais com a magnitude da anisotropia pré-operatória do que com a magnitude da transposição efetuada. Especula-se que estes fatores coadjuvantes possam ser, em alguns casos, de natureza inervacional. Scott ${ }^{9}$ descreveu um caso de exotropia em V sem disfunção de oblíquos, em que demonstrou, eletromiograficamente, pela primeira vez, inervação anormal para o reto lateral em supraversão.

Cabe ressaltar crítica ao método empregado para a mensuração da anisotropia. Não há uniformidade ou padronização na quantidade de extensão e flexão da cabeça e, possivelmente, essa técnica poderia estimular receptores labirínticos ou proprioceptivos cervicais, alterando a medida. O perímetro com arco de 90 graus e uma opção adotada por alguns autores ${ }^{13}$ para a medida da anisotropia.

O fracasso na obtenção de equação envolvendo as três variáveis sugere presença de outra(s) não identificada(s) ou a existência de modelos matemáticos não lineares que as correlacione adequadamente.
Em suma, concluímos que a transposição vertical e simétrica dos músculos retos horizontais é eficaz para o tratamento de anisotropias verticais sem a correspondente disfunção de músculos oblíquos, sendo este resultado estável em seguimento prolongado. Os pacientes apresentaram, na nossa amostra, predominância de formas em A $(81,4 \%)$ e a magnitude da anisotropia foi, em média, de aproximadamente 19 DP. Nas formas em A, a correção da anisotropia correlacionou-se intensamente com a magnitude da transposição efetuada e com a magnitude da anisotropia pré-operatória, sobretudo com esta, sugerindo, assim, ser o procedimento auto-ajustável. Não foi possível elaborar equação que correlacionasse magnitude da anisotropia pré-operatória, magnitude da transposição e correção da anisotropia. As mesmas observações não puderam ser feitas em relação à incomitância em $\mathrm{V}$ devido à insuficiência da amostra. A razão disto é a raridade de casos de anisotropias alfabéticas sem hiperfunção de oblíquos que exigem correção cirúrgica.

\section{SUMMARY}

Purpose: Vertical transpositions of the horizontal rectus muscles is the preferred operation in cases of $A$ and $V$ pattern strabismus in which oblique muscle dysfunction is inadequate to allow oblique surgery. The purpose of this study was to assess the results of performed transpositions and to point out factors that influence outcome so as to establish an equation correlating magnitude of transposition to be performed for a given pattern size.

Methods: 43 patients with nonparetic horizontal strabismus associated with $A$ or $V$ pattern, without oblique muscle dysfunction, who underwent bilateral and symmetrical vertical transpositions of the horizontal rectus muscles, were retrospectively studied.

Results: $81.4 \%$ of patients had esotropia of A pattern. The mean pattern size was 19 PD and most transpositions were $4 \mathrm{~mm}$ shifts, with a success rate of 66.7 to $79.5 \%$, that is, residual A pattern up to 5 PD or $V$ pattern up to 10 PD. The pattern correction is closely related to the preoperative pattern size and with the magnitude of transpostion carried out, mostly with the former, therefore suggesting that the procedure is self-adjustable. The linear equation with 3 variables was clinically incoherent and was, therefore, abandoned.

Conclusions: Symmetrical vertical transpositions of the horizontal rectus muscles is a effective long term procedure for collapsing $A$ and $V$ pattern, whenever oblique muscle dysfunction is absent.

Keywords: Transposition; $A$ and $V$ pattern.

\section{REFERÊNCIAS BIBLIOGRÁFICAS}

1. Urist M. Recession and upward displacement of the medial rectus muscles in A - pattern esotropia. Am J Ophthalmol 1968;65:769-73. 
2. Brown HW. Vertical deviations. Em Strabismus Symposium. Tr Am Acad Ophthal Otolaryngol 1953;57:157-9.

3. Urrets-Zavalia Jr. A. Significance of congenital cyclovertical motor defects of the eyes. Brit J Ophthalmol 1955;39:11-20.

4. Knapp P. Vertically incomitant horizontal strabismus. The so-called A and V syndromes. Trans Am Ophthal Soc 1959;57:666-98.

5. von Noorden GK, Burian HM. Binocular vision and ocular motility. St. Louis, The C.V. Mosby Co 1974;337-40.

6. Goldstein JH. Monocular vertical displacement of the horizontal rectus muscles in the A and V patterns. Am J Ophthalmol 1967;64:265-7.

7. Villaseca A. Results of horizontal muscle surgery in the A and V syndromes. J Ped Ophthalmol 1965;2:19-36.

8. Almeida HC, Pereira Real M. Anisotropias alfabéticas. Comunicação ao VIII Congresso Brasileiro de Prevenção de Cegueira, Rio de Janeiro, 1988

9. Scott AB. A and V patterns in exotropia. An electromyographic study of horizontal rectus muscle. Am J Ophthalmol 1968;65:12-14.

10. Scott WE, Drummond GT, Keech RV. Vertical offsets of horizontal recti muscles in the management of $\mathrm{A}$ and $\mathrm{V}$ pattern strabismus. Aust $\mathrm{N} \mathrm{Z} \mathrm{J}$ Ophthalmol 1989;17(3):281-8.
11. Delmonte, MA \& cols. Vertical shift of the medial recti in A-pattern esotropia Report of the 7th Quadrennial Meeting of the International Strabismological Association (ISA), Vancouver 1994;291-300.

12. Miller JM, Demer JL, Rosenbaum AL. Effect of transposition surgery on rectus muscle paths by magnetic resonance imaging. Ophthalmology 1993;100(4):475-87.

13. Almeida HC. Correção cirúrgica das anisotropias em A e V. Tese de Livre Docência. Apresentada ao Depto. de Oftalmologia da Faculdade de Medicina da UFMG. Belo Horizonte, 1978 .

14. Prieto-Díaz J, Souza-Dias C. Estrabismo., Segunda ed. São Paulo: Roca, $1986 ; 233-60$

15. Souza-Dias C, Almeida HC. Estrabismo. Conselho Brasileiro de Oftalmologia, São Paulo: Roca 1993;102 e 162-8.

16. Arthur B. Proceedings of the American Academy of Ophthalmology, San Francisco, 1994.

17. Bicas HEA. Mecanismos de ação das transposições de músculos retos para a correção de incomitâncias em A ou V. Boletim do Conselho Latino-Americano de Estrabismo (CLADE) 1994;(8):12-4.

18. Postic A, von Noorden GK, In: Burian HM. Binocular Vision and ocular motility. St. Louis: C.V. Mosby Co., 1974;338.

\title{
SIMPÓSIO INTERNACIONAL DA SOCIEDADE BRASILEIRA DE CIRURGIA PLÁSTICA OCULAR
}

\author{
7 a 9 de Dezembro de 2000 \\ OTON PALACE HOTEL \\ Belo Horizonte-MG
}

Informações: Consult Comunicação e Marketing

Tel./Fax: (31) 274-1550

e-mail: comunica@consultcom.com.br

internet: www.consultcom.com.br 